\title{
UNA ESTRATEGIA METACOGNITIVA Y DE AUTORREGULACIÓN EN LA RESOLUCIÓN DE PROBLEMAS EN FÍSICA ${ }^{+}$
}

\author{
María Rosa Suárez Vargas \\ Facultad de Ingeniería \\ Universidad Nacional de Jujuy \\ San Salvador de Jujuy - Argentina \\ Leonor Colombo de Cudmani \\ Instituto de Física - Facultad de Ciencias Exactas \\ Universidad Nacional de Tucumán \\ Tucumán - Argentina
}

\section{Resumen}

Este estudio es parte de una investigación más amplia cuyo propósito es el diseño de estrategias curriculares de evaluación del aprendizaje con énfasis en procesos metacognitivos y de autorregulación (MC y AR), superando la concepción reduccionista de evaluación como sinónimo de acreditación. En este trabajo se analiza la importancia de la metacognición y la autorregulación en el aprendizaje de la Física y en su evaluación. Se caracteriza un marco referencial que permita derivar criterios que guien las actividades de enseñanza y aprendizaje de estos procesos y se diseña y evalúa una estrategia de aula para ser implementada con estudiantes de Física de nivel medio. El análisis sistemático de los instrumentos de recolección de datos propuestos muestra que se ajusta a los objetivos y al modelo teórico que guía la investigación. La aplicación de esta estrategia en la exploración de campo aporta indicios para suponer que este instrumento ayuda a los estudiantes a reflexionar sobre sus propias dificultades en la resolu-

\footnotetext{
+ A metacognitive strategy and self-regulation processes in solving Physics problems

* Recebido: dezembro de 2008. Aceito: agosto de 2009.
} 
ción de problemas y a buscar modos de superarlas. Revaloriza la función de la evaluación permitiendo al docente conocer las dificultades de aprendizaje para retroalimentar el proceso de enseñanza.

Palabras-clave: Evaluación; estrategias evaluativas; metacognición; resolución de problemas.

\begin{abstract}
This work is part of a wider research which aims at the design of curricular strategies regarding learning evaluation focusing in metacognitive and self-regulation processes, thus widening the idea that an evaluation is just a synonym for a "passing score". In this work, the importance of metacognition and self-regulation of learning and evaluation of Physics will be analyzed. A frame of reference will be developed to create criteria to be used as a guide for learning and teaching activities. What is more, a classroom strategy will be created to work with students of High School. The systematic analysis of the proposed data collection instruments shows that it fits the objectives and the theoretical model that guides the research. The implementation of this strategy, gives some evidence to suppose that this instrument helps the students not only to find ways to solve problems but also to reflex on their own difficulties during the process of resolution. Moreover, this strategy revalues the function of the evaluation and let the teacher knows the difficulties of the learning process in order to feedback the teaching process.
\end{abstract}

Keywords: Evaluation; evaluation strategies; metacognition; solving problems.

\title{
I. Introducción
}

La reflexión crítica sobre nuestra propia práctica docente nos permite inferir que la evaluación del aprendizaje es, en general, sinónimo de examen, medición y acreditación. Se trata de una concepción reduccionista muy generalizada en la práctica docente en diferentes contextos, como lo muestran numerosos trabajos. 
(PEREZ, 1993; RAYA, 2001; VILLALONGA, 2002; CUDMANI, 2003). En general se trabaja con una concepción de evaluación cuantitativa, no formativa que consiste, en mayor medida, en la aplicación de pruebas para obtener información a partir de las que se asignan calificaciones a los alumnos, a fin de decidir su promoción. Por cierto que esta práctica no es compatible con los modelos de aprendizaje estructuralistas que propone actualmente la investigación educativa en ciencias. Definiremos más adelante un modelo de evaluación superador que se adecue mejor a los modelos mencionados.

En este trabajo se enfatiza que el logro más importante en la enseñanza es la adquisición de la autonomía en el aprendizaje:

... el aprendizaje de las ciencias no puede limitarse a realizar cambios hacia paradigmas preestablecidos y seleccionados por el docente, sino que debe intentar desarrollar estrategias que permitan al estudiante modificar sus paradigmas, sus esquemas interpretativos en forma autónoma, autogenerada, cada vez que lo requieran las situaciones problemáticas a las que se enfrenta... en algún momento él (el estudiante) deberá desarrollar sus propias estrategias para detectar y enfrentar estos conflictos. Será necesario que adquiera la capacidad de cuestionarse, de detectar lagunas, incoherencias, contradicciones, paradojas en su estructura cognitiva (CUDMANI et al, 1991, p. 237-238).

Se deberían planificar propuestas innovadoras en la enseñanza de la Física que sean más eficientes para lograr un aprendizaje significativo, que ocurre cuando la nueva información se relaciona con conceptos o proposiciones relevantes preexistentes y el almacenamiento de la información se da formando una jerarquía conceptual, en la que los elementos más específicos de conocimientos son ligados (y asimilados) con conceptos más generales y más inclusivos (AUSUBEL, 1978) y para ello en particular nos proponemos indagar los aportes que pueden derivar de la autoevaluación y de la metacognición.

Entendemos por autoevaluación la revisión global y sistemática por parte del estudiante de sus procesos de aprendizaje., por metacognición la planificación y supervisión-revisión de sus procesos de aprendizaje y por meta evaluación el análisis del proceso de evaluación, la evaluación de la evaluación.

Así, la meta de este trabajo es el análisis, diseño y posterior aplicación de una estrategia de evaluación del aprendizaje con énfasis en procesos metacognitivos. Las estrategias de "Aprendizaje Basado en resolución de Problemas" (ABP), 
han sido muy valorizadas y sus potencialidades analizadas por diversos autores en la investigación sobre enseñanza de la Física (CUDMANI, 1998; COSTA; MOREIRA, 2001; HENK, 1989; AIZICZON; CUDMANI, 2006). Sin embargo no siempre se orientó al desarrollo de la autorregulación y la metaevaluación del aprendizaje. Nos proponemos, en el contexto de la enseñanza de la Física para estudiantes de nivel medio, incorporar ítems para incluir esta actividad.

Seguidamente, se definen las líneas principales del marco teórico en que se encuadra la propuesta que incorpora elementos de metacognición y autoevaluación y se analizan algunos aspectos importantes que sirven de base para el diseño y la realización de este trabajo.

\section{Fundamentos teóricos}

\section{II.1 El lugar de la evaluación en el proceso de enseñanza-aprendizaje}

Sin lugar a dudas, la evaluación constituye un elemento fundamental en el proceso de enseñanza-aprendizaje. Es utilizada de diferentes maneras, el sistema educativo promociona a un estudiante a través de esta instancia, el docente la utiliza, en algunos casos, para mantener la disciplina académica, también para descubrir qué alumnos y en qué medida han conseguido alcanzar las metas propuestas inicialmente, y ocasionalmente puede se usada para indicar la eficacia de ciertas estrategias de enseñanza y entonces constituye un elemento valioso para la toma de decisiones por parte del profesor (retroalimentación).

Uno de los posibles efectos que producen las evaluaciones para los estudiantes es que "moldean e influyen en el desarrollo de destrezas y estilos de aprendizaje de los alumnos" (CROOKS, 1988, p. 443)

Los principios evaluativos de este trabajo se basan en aportes de teorías de aprendizaje que han mostrado ser eficientes en la enseñanza de las disciplinas científicas. Las teorías consideradas fueron: Psicogenética de Piaget, de Aprendizaje Significativo de Ausubel (1978) y el Enfoque Histórico Cultural, con la Teoría de la Actividad y la Teoría de Formación por Etapas de las Acciones Mentales de Vigotsky, Leontiev, Galperin y otros (SANZ, 1991; MOREIRA, 1997a; MOREIRA, 1997b; PÉREZ, 2000). Todas estas cuestiones fueron tratadas con mayor profundidad en los trabajos sobre evaluación de Raya (2001) y Colombo de Cudmani et al (2003), cuyos consensos más importantes pueden resumirse en los siguientes:

- Aprendizaje se entiende como una construcción (reconstrucción y coconstrucción) de significados. 
- La concepción del conocimiento científico se entiende como un proceso abierto, dinámico, en permanente construcción.

- Se valora la importancia para el aprendizaje del grado de desarrollo de la estructura cognoscitiva de los alumnos.

- Se considera la importancia de tener en cuenta lo que "ya se sabe" (ideas previas, preconcepciones).

- La necesidad de considerar la complejidad del campo cognitivo, el que no se reduce a lo conceptual sino, que se integra con modos de razonamientos, estrategias de aprendizajes, fines, valores, intereses, concepciones epistemológicas y ontológicas.

- El reconocimiento de la dimensión social, al entender el conocimiento científico como construcción social y valorizando la búsqueda de conceptos, el trabajo grupal, la comunicación, entre otros.

- Los conceptos y proposiciones adquieren significados más objetivos y precisos dentro de un contexto lógico, epistemológico o psicológico. Los enunciados observables usan siempre el contexto de alguna teoría, explícita o no.

Cabe destacar que estos consensos han sido seleccionados como marco referencial por el proyecto de Investigación "Transferencia de resultados de investigación en la superación de dificultades en el aprendizaje de conceptos y procedimientos en ciencia y tecnología" del Consejo de Investigaciones de la Universidad Nacional de Tucumán, dentro del cual se integra esta indagación.

En referencia al objetivo de este trabajo profundizaremos en el marco teórico referido específicamente a la Metacognición:

De acuerdo a la concepción de Flavell, consideraremos que:

La metacognición se refiere al conocimiento que uno tiene sobre los propios procesos y productos cognitivos o sobre cualquier cosa relacionada con ellos, es decir, las propiedades de la información o los datos relevantes para el aprendizaje. Por ejemplo, estoy implicado en metacognición (metamemoria, metaaprendizaje, metaatención, metalenguaje, etc.) si me doy cuenta de que tengo más problemas al aprender A que al aprender B, si me ocurre que debo comprobar $C$ antes de aceptarlo como un hecho... La metacognición se refiere, entre otras cosas, al control y la orquestación y regulación subsiguiente de estos procesos (FLAVELL, 1976, p. 232). 
La metacognición puede estar presente en diversos aspectos del proceso de enseñanza aprendizaje como por ejemplo en la resolución de problemas, en las concepciones epistemológicas de los alumnos, en el cambio conceptual, en los criterios de comprensión y explicación que utilizan los alumnos, en la formulación de preguntas y la motivación (CAMPANARIO; CUERVA; MOYA; OTERO, 1998).

Si bien los conceptos de metacognición y autorregulación están íntimamente relacionados, es importante diferenciarlos, el primer término se refiere al grado de conocimiento que el estudiante tiene sobre sí mismo, sobre los requerimientos de la tarea y sobre el propio proceso de aprendizaje que se está produciendo, supone que posea una buena dosis de conciencia y de reflexión respecto a esos aspectos; la autorregulación supone una actividad de planificación y supervisiónrevisión de la tarea para lograr superar las dificultades de aprendizaje que ya ha detectado.

Para este trabajo se ha seleccionado, de entre aquellas actividades con que se trabaja habitualmente en Física para construir sus contenidos (conceptuales, metodológicos y actitudinales), las que se relacionan con la resolución de problemas para incluir en ellas los aspectos referidos a la autorregulación del aprendizaje.

\section{II.2 La metacognición en la resolución de problemas}

Se trata de que el docente de ciencias utilice recursos, que tal vez ya le sean conocidos, pero ahora rescatando y poniendo énfasis en el desarrollo de capacidades que favorezcan la metacognición y la autoevaluación (proceso que se inicia en el propio individuo que consiste en una revisión global y sistemática de sus procesos de aprendizaje) y para que esto sea posible deben ser compatibles o incidir en algunos de los siguientes aspectos (CAMPANARIO, 2000):

a) Conocimiento o control del propio conocimiento (conocimiento espontáneo y científico) o procesos cognitivos (estrategias de pensamiento y estrategias de aprendizaje).

b) Autorregulación cognitiva, incluyendo el control del estado actual de la propia comprensión.

c) Detección y análisis explícito, por el propio estudiante, de las barreras u obstáculos epistemológicos que frenan el aprendizaje. 
d) Desarrollo de la capacidad de detectar cuestiones referidas a la estructura, producción y organización del conocimiento, incluyendo las relaciones entre partes aparentemente diferenciadas de un área determinada.

Utilizando problemas tradicionales, los que se usan habitualmente, se consigue la mayoría de las veces que los estudiantes desarrollen una metodología superficial y mecánica lo que puede conducirlos a obtener una solución incorrecta. El objetivo de esta mecánica consiste en hacer reflexionar a los alumnos sobre los errores que cometen cuando se enfrentan a los problemas de una manera mecanicista y superficial. Al estar presente la reflexión sobre sus propio aprendizaje el estudiante aprende a aprender y desarrolla capacidades metacognitivas.

\section{II.3 La metacognición como facilitadora en la resolución de problemas}

Las habilidades metacognitivas tienen un papel importante en la resolución de problemas ya que ayudan a (DAVIDSON; STERNBERG, 1998)

a) Codificar estratégicamente la naturaleza del problema y obtener una representación mental de sus elementos.

b) Seleccionar las estrategias adecuadas para la consecución del objetivo

c) Identificar los obstáculos que impiden y dificultan el progreso.

"El hecho de conocer la propia cognición no indica que automáticamente se utilicen procesos metacognitivos" (ROBERTS, 1993, p. 259). Una persona puede ser conciente de que no entiende un problema pero no llevar a cabo ninguna estrategia para superar su dificultad. Hay una independencia entre el conocimiento de la metacognición y la aplicación de la metacognición.

Se observan dos aspectos relacionados con la metacognición que permiten una resolución más acertada de un problema como es la verbalización a lo largo de la resolución del problema y las experiencias metacognitivas.

En cuanto a la verbalización, se ha probado que puede favorecer los mecanismos metacognitivos ya que permite ser más consciente de las estrategias de resolución, de las dificultades del problema y la monitorización del proceso.

Numerosos estudios sobre resolución de problemas indican que mediante la técnica de "pensamiento en voz alta" se puede estudiar con detenimiento todos los procesos subyacentes a la resolución del problema facilita a obtención de la respuesta exitosa del problema. Aunque es el tipo de pensamiento implicado en la resolución de problemas lo que provoca una modificación en la resolu- 
ción y no el pensamiento en voz alta por sí mismo (ERICSSON; SIMON 1980, p. 215).

Las experiencias metacognitivas están altamente relacionadas con la resolución de problemas; son los sentimientos y sensaciones que experimenta el sujeto al inicio, durante o al final de la resolución de un problema. Estas pueden afectar los objetivos del problema, al conocimiento metacognitivo y a las estrategias utilizadas durante el proceso de resolución (FLAVELL, 1979, p. 906).

\section{Metodología}

\section{III.1 Diseño de un instrumento para una primera indagación exploratoria de este campo de investigación}

Del marco teórico enunciado se desprende que aunque el docente de ciencias utilice recursos que tal vez ya le sean conocidos, deben tener una explicita intención de incentivar la MC y la AR.

Frente a la necesidad de generar instrumentos que nos aporten información experimental sobre este problema, se realizó una extensa revisión bibliográfica en revistas especializadas encontrando escasos ejemplos de actividades de aula, aplicadas a la Física, que persigan estos objetivos.

Intentamos por ello, elaborar una propuesta que permitiera concretar estas ideas en actividades de aula, en una primera indagación exploratoria

Se diseñó, para ello, actividades en las cuales, en la resolución de situaciones problemáticas para evaluar el aprendizaje, sobre un tema concreto: aplicación de las leyes de la Dinámica, se incluía un cuestionario para indagar explícitamente sobre metaevaluación y autoregulación. Se espera que, en caso de probar su eficacia, éste pueda ser tomado como modelo para elaborar cuestionarios análogos en otras temáticas. En esta primera instancia el cuestionario deberá ser respondido por escrito individualmente. El apéndice I reproduce estas actividades.

De los fundamentos teóricos, de la situación contextual y los objetivos de este trabajo se desprenden los criterios considerados para la elaboración del cuestionario:

- La función de la evaluación

La evaluación es utilizada como herramienta metacognitiva y constituye un elemento valioso para la toma de decisiones por parte del profesor para realimentar el proceso de enseñanza aprendizaje y para el estudiante a los efectos de 
reflexionar sobre su proceso de aprendizaje, sus dificultades y modos de superarlas.

- La utilización de problemas tradicionales donde se promueva la reflexión.

La utilización de problemas tradicionales acompañados de un cuestionario nos permite enfrentar a los estudiantes con situaciones que se usan habitualmente en las clases de Física y al estar presente la reflexión sobre sus propias dificultades se propicia el desarrollo de las capacidades metacognitivas.

La identificación de sus obstáculos de aprendizaje permite hacer conscientes sus dificultades.

La búsqueda de estrategias que les permita superar sus dificultades constituye un elemento valioso para lograr la autonomía en el aprendizaje.

Se espera que, en caso de probar su eficacia, éstos puedan ser tomados como modelo para elaborar cuestionarios análogos en otras temáticas. En esta primera instancia el cuestionario deberá ser respondido por escrito e individualmente. Posteriormente y en base de análisis de los datos obtenidos, se indagarán las categorías investigadas usando la técnica de Entrevista semiestructurada a una muestra, aleatoria pero menos numerosa, de alumnos. Con estos resultados se controlará la validez de la encuesta y la saturación de las categorías

\section{III.2Aplicación del instrumento diseñado}

Uno de los autores de este trabajo fue el responsable de implementar esta propuesta puesto que la experiencia debía ser implementada durante el desarrollo de los cursos normales de la materia, el contenido se seleccionó sobre la base de su factibilidad, respetando los cronogramas vigentes. Resultó conveniente abordar el tema de Dinámica en un plano inclinado.

Para la experiencia se seleccionó un grupo de 34 estudiantes que integran un curso normal de clases. Dichos jóvenes asisten a un colegio secundario de la provincia de Jujuy y cursan el quinto año del bachillerato común y tienen en promedio una edad de 16 años.

La enseñanza del tema se desarrolló con clases teórico prácticas, donde se resolvieron situaciones problemáticas conceptuales y de aplicación de las leyes de la dinámica, tratando de ajustar la metodología de enseñanza a los consensos constructivistas que se explicitan en el marco teórico (p. 517) y la aplicación del instrumento diseñado se realizó al finalizar el desarrollo del tema, a modo de cierre. 
Del análisis de las respuestas a este cuestionario y teniendo en cuenta los modelos de aprendizaje y de evaluación propuestos, los docentes investigadores definen sus categorías y dimensiones de análisis para la evaluar la propuesta innovadora.

\section{Análisis de los resultados}

Para el análisis de datos se establecieron categorías y dimensiones que surgieron de categorías "a priori" que resultan de los objetivos y del marco teórico explicitado, en base a las cuales los docentes diseñaron la estrategia.

Las categorías I y II se proponen indagar en que medida el alumno reflexiona y controla su propio conocimiento y las dificultades que perturban sus procesos cognitivos y sus estrategias de pensamiento y de aprendizaje)

La categoría III indaga obre proceso de Autorregulación cognitiva, incluyendo el control del estado actual de la propia comprensión y las estrategias a que recurre para superar sus dificultades.

La categoría IV permite rescatar las voces de los alumnos en lo que se refiere al juicio que les mereció la nueva estrategia, proporcionando a los docentes importantes elementos de juicio para realimentar sus estrategias de aula.

La Tabla 1 muestra una síntesis de categorías y dimensiones establecidas para el análisis.

Tabla 1 - Categorías y dimensiones de análisis.

\begin{tabular}{|l|l|}
\hline \multicolumn{1}{|c|}{ Categorías } & \multicolumn{1}{|c|}{ Dimensiones } \\
\hline $\begin{array}{l}\text { I - Dificultad en la resolución } \\
\text { (determina si hubo o no dificultad } \\
\text { en la resolución de la situación } \\
\text { planteada) }\end{array}$ & $\begin{array}{l}\text { A- El estudiante SI tuvo dificultad en la } \\
\text { resolución del problema propuesto. } \\
\text { resolución del problema propuesto. }\end{array}$ \\
\hline $\begin{array}{l}\text { II - Causas de la dificultad (se } \\
\text { identifican las posibles causas de la }\end{array}$ & A- La interpretación del enunciado \\
dificultad) & C- La identificación de los datos \\
& actúan sobre el cuerpo de las fuerzas que \\
& D- La aplicación de las leyes de la Dinámi- \\
& ca \\
& E- El planteo de las ecuaciones \\
& F- La resolución de las ecuaciones \\
G- La influencia del rozamiento
\end{tabular}

Vargas, M. R. S. e Cudmani, L. C. 


\begin{tabular}{|l|l|}
\hline $\begin{array}{l}\text { III - Estrategias superadoras (son } \\
\text { algunas de las estrategias que le }\end{array}$ & A- Pedir ayuda a un compañero \\
permitirán al estudiante superar su & Bolicitar una nueva explicación al profe- \\
dificultad) & C- Resolver mayor cantidad de ejercicios \\
& D- Cambiar la forma de estudiar \\
& No sabe como superar su dificultad. \\
\hline $\begin{array}{l}\text { IV - Valoración del instrumento (el } \\
\text { estudiante emite un juicio sobre la } \\
\text { utilidad del cuestionario como } \\
\text { herramienta metacognitiva) }\end{array}$ & A- El cuestionario propuesto es útil como \\
herramienta metacognitiva. \\
B - El cuestionario propuesto no promueve \\
\hline
\end{tabular}

En I la situación planteada generó dificultad en el 79\% de los estudiantes. Sólo el $21 \%$ manifestó no tener inconveniente en resolver el problema

La tabla 2, 3 y 4 registran los porcentajes de alumnos que se encuadran en cada item de las otras categorías.

Tabla 2 - Dificultad en la resolución.

\begin{tabular}{|l|c|}
\hline A- Interpretación del enunciado & $22 \%$ \\
\hline B- Identificación de los datos & $15 \%$ \\
\hline C- Identificación de las fuerzas que actúan sobre el cuerpo & $22 \%$ \\
\hline D- Aplicación de las leyes de la Dinámica & $26 \%$ \\
\hline E- Planteo de las ecuaciones & $74 \%$ \\
\hline F- Resolución de las ecuaciones & $7 \%$ \\
\hline G- La influencia del rozamiento & $7 \%$ \\
\hline
\end{tabular}

(Los porcentajes están expresados en función del total de alumnos que encontraron dificultad en la resolución de la situación planteada).

En II los resultados obtenidos que se detallan en la tabla muestran que la mayor dificultad se presenta en el planteo de las ecuaciones. Esta toma de conciencia podría ser importante para que los alumnos rescaten el valor de sus conocimientos matemáticos en la resolución de problemas.

La influencia del rozamiento se pone de manifiesto al comparar las dos situaciones planteadas a los estudiantes. Contra lo que podría esperarse, para los alumnos esta nueva complejidad no es determinante para dificultar la resolución de la situación planteada. Es posible que los problemas planteados en las actividades 
no sean eficientes para sacar a luz las dificultades conceptuales del fenómeno de roce. Parece un tema interesante para una nueva exploración.

Tabla 3 - Estrategias superadoras de las dificultades.

\begin{tabular}{|c|cc|}
\hline A- Pedir ayuda a un compañero & \multicolumn{2}{|c|}{44} \\
\hline B- Solicitar una nueva explicación al profesor & $\%$ & 41 \\
\hline I. C- Resolver mayor cantidad de ejercicios & $\%$ & 67 \\
\hline D- Cambiar la forma de estudiar & $\%$ & 41 \\
\hline E- No sabe como superar su dificultad & $\%$ & 7 \\
\hline
\end{tabular}

(Los porcentajes están expresados en función del total de alumnos que encontraron dificultad en la resolución de la situación planteada).

De su lectura se deduce que la mayoría de los estudiantes consideran que resolver mayor cantidad de ejercicios es lo que en mayor medida puede favorecer su aprendizaje. Es notable también que pese a que se espera que el alumno priorice fuertemente su interacción con el docente (41\%) para tratar de superar sus dificultades, atribuyen tanto o más valor $(44 \%)$ a la interacción con sus pares. Esto se podría interpretar en el sentido de que ellos son concientes de la importancia del grupo en su aprendizaje, tal como lo postula nuestro modelo.

El 41\% que destaca la necesidad de Cambiar la forma de estudiar nos sugiere otro aspecto para profundizar. Parece interesante indagar sobre cuales son sus propuestas fundadas para mejorar.

Tabla 4 - valoración del instrumento por parte del estudiante.

\begin{tabular}{|l|c|}
\hline A- El cuestionario propuesto es útil como herramienta metacognitiva & $88 \%$ \\
\hline B- El cuestionario propuesto no promueve la metacognición & $22 \%$ \\
\hline
\end{tabular}

EL $88 \%$ de los estudiantes valoraron positivamente el uso del cuestionario para ayudarlos a reflexionar sobre sus dificultades y modos de superarlas.

Manifestaron la utilidad de este instrumento en dos sentidos, para uso propio en cuanto pueden identificar sus dificultades, como también para uso del 
profesor, que a partir del análisis del mismo puede detectar como regular el proceso de enseñanza aprendizaje conociendo donde consideran los estudiantes que se encuentran con obstáculos para la solución de problemas y cómo ven posibles soluciones.

\section{Otras categorías de análisis que surgen del campo explorado}

La lectura y relectura de las respuestas al cuestionario sugiere la conveniencia de incluir otras categorías de análisis tales como:

La dificultad en la resolución de la situación se debe a:

- Falta de estudio

- Ausencia a clase

Para superar la dificultad el estudiante cree que debe:

- Consultar bibliografía.

- Aprovechar mejor sus tiempos

- Prestar mayor atención en clase.

Se espera profundizar en la interpretación de estos resultados utilizando otros instrumentos de investigación como la entrevista grupal semiestructurada. Así, en otra instancia posterior, las respuestas serán analizadas en grupo de tres alumnos y los docentes actuando como investigadores participantes ocasión en que los estudiantes deberán " verbalizar " sus procesos de aprendizaje y argumentar para defender sus modos de razonar y sus conclusiones ante sus compañeros y ante el docente y podrá además relatar sus experiencias en cuanto a sentimientos y sensaciones vividas durante el proceso.

El docente investigador, como observador participante podrá registrar datos en términos de sus categorías de análisis para la evaluar la eficacia de la estrategia (metodología de investigación) y sacar sus conclusiones para realimentar el proceso de aprendizaje (metodología de enseñanza).

\section{Primeras conclusiones}

- Se plantea en este trabajo un problema para indagar: la generación de estrategias de aula eficientes para desarrollar los procesos de MC y AE y se fundamenta su importancia para el aprendizaje significativo y la enseñanza de la Física con el propósito de favorecer la autorregulación

- Se explicita un marco de referenciales teóricos para fundamentar significados e importancia de estos procesos de autorregulación dentro de un modelo de 
aprendizaje que responde a los consensos de las teorías estructuralistas del aprendizaje

- Se elaboran criterios derivados de los marcos teórico y contextuales y de los objetivos los cuales sirven de guía en el diseño estrategias de aula eficientes para desarrollar estas capacidades.

- Fundamentados en esos criterios, se diseñan una serie de cuestionarios, contextualizados en la enseñanza de la Física para la enseñanza media (pero que se espera puedan servir de modelos para otros contextos disciplinares e institucionales) para recoger datos experimentales sobre la problemática.

- Se valida la encuesta en base de criterios internos derivados del modelo, del contexto y de los objetivos

El análisis sistemático de los instrumentos de recolección de datos propuestos muestra que se ajusta a los objetivos y al modelo teórico que guía la investigación.

La aplicación de esta estrategia en la exploración de campo permite suponer que este tipo de instrumento favorece el desarrollo de la capacidad metacognitiva en los estudiantes, les permite reflexionar sobre sus propias dificultades en la resolución de una situación propuesta y buscar el modo de superarlas. También revaloriza la función de la evaluación permitiendo al docente conocer las dificultades de aprendizaje de sus estudiantes y de esta manera utilizar el instrumento para retroalimentar el proceso de enseñanza aprendizaje.

En efecto, este trabajo es la etapa previa y exploratoria de un estudio más amplio donde se pondrán en práctica otros instrumentos tendientes a desarrollar las capacidades metacognitivas de los estudiantes para favorecer su autonomía en el aprendizaje.

Posteriormente se analizará la posibilidad de extender estas estrategias a otras áreas de la disciplina, en otros contextos y niveles educativos además de procurar evaluarlas respecto a su eficiencia para desarrollar las capacidades metacognitivas en los estudiantes y para favorecer su autonomía en el aprendizaje con otros instrumentos de recolección de datos.

\section{Referencias bibliográficas}

AIZICZON, B.; CUDMANI, L. C. La evaluación del aprendizaje significativo en el ABP en biofísica en Medicina, las voces de los alumnos. In: CONFERENCIA INTERAMERICANA SOBRE EDUCACIÓN EN LA FÍSICA, IX, 2006, San José, Costa Rica. Memorias... ISBN 978-9968-9939-9-9. 
ALONSO, M.; PEREZ, D. G.; TORREGOSA, J. M. Los exámenes de Física en la enseñanza por transmisión y en la enseñanza por investigación. Enseñanza de las Ciencias, v. 10, n. 2, p.127-138, 1992.

AUSUBEL, D. Psicología Educativa. México: Trillas, 1978.

BAKER, L. Metacognition, reading and science education. In: SANTA, C. M.; ALVERMANN, D. (Eds.) Science learning: Processes and applications, Newark: International Reading Association, 1991.

BERTONI, A.; POGGI, M.; TEOBALDO, M. Evaluación: Nuevos significados para una práctica compleja. Buenos Aires: Ed. Kapeluzs, 1996.

CAMPANARIO, J. M. El desarrollo de la metacognición en el aprendizaje de las ciencias: estrategias para el profesor y actividades orientadas al alumno. Revista de Enseñanza de las Ciencias, v. 18, n. 3, p. 369-380, 2000.

CAMPANARIO, J. M.; CUERVA, J.; MOYA, A.; OTERO, J. C. La metacognición y el aprendizaje de las ciencias. In: BANET, E.; PRO, A. de (Eds.). Investigación e innovación en la enseñanza de las ciencias. Murcia: Ed. DM, 1998. v. I, p. $36-44$

COSTA S. C. da; MOREIRA, M. A. A resolução de problemas como um tipo especial de aprendizagem significativa. Caderno Catarinense de Ensino de Física, v. 18, n. 3, p. 263-277, dez. 2001.

CROOKS, T. J. The impact of classroom evaluation practices on students. Review of Educational Research, v. 58, n. 4, p. 438-481, 1988.

CUDMANI, L. C. Resolución de problemas en el aula. Revista de Ensino de Física, v. 20, n. 3, 1998.

CUDMANI, L. C.; GARCÍA, P. V.; RAYA, F. Marcos teóricos de referencia para orientar la evaluación del aprendizaje en cursos básicos universitarios en ciencias. In: CONFERENCIA INTERAMERICANA SOBRE EDUCACIÓN EN LA FÍSICA, VIII, 2003, La Habana, Cuba. Memorias... (CD-ROM)

CUDMANI, L. C.; PESA, M.; SANDOVAL, J. S. La generación autónoma de conflictos cognoscitivos para favorecer cambios de paradigmas en el aprendizaje de la Física. Revista de Enseñanza de las Ciencias, v. 9, n. 3, p. 237-242, 1991. 
DAVIDSON, J. E.; STERNBERG, R. J. Smart problem solving: how metacognition helps. In: HACKER, D. J.; DUNLOSKY, J.; GRAESSER, A. C. (Eds.). Metacognition in educational theory and practice. Mahwah, New Jersey: LEA, 1998. p. 47-68.

DOMÉNECH, A. M. El papel de la inteligencia y de la metacognición en la resolución de problemas. 2004. Tésis (Doctoral) - Universitat Rovira I Virgili, Tarragona.

ERICSSON, K. A.; SIMON, H. A. Verbal report as data. Psychological Review, v. 87, n. 3 , p. $215-251,1980$.

FLAVELL, J. H. Metacognition and cognitive monitoring: a new area of cognitive-developmental inquiry. American Psychologist, v. 34, n. 10, p. 906911, 1979.

FLAVELL, J. H. Metacognitive aspects of problem solving. In: RESNICK, L. B. The nature of intelligence. Hillsdale, New Jersey. Lawrence Erimbaum, 1976.

GARCÍA, P. V. Un enfoque alternativo para la evaluación del Cálculo en una Facultad de Ciencias. 2003. Tesis (Magíster) - Universidad Nacional de Tucumán, Tucumán, Argentina.

GARCÍA, P. V.; CUDMANI, L. C. Algunas patologías evaluativas en el aula de Matemática. Un estudio exploratorio. Enviado a revista Heuresis, junio de 2005.

GARCÍA, P. V.; CUDMANI, L. C. La evaluación del aprendizaje en cursos básicos de Cálculo en una Facultad de Ciencias (Segunda parte) In: SIMPOSIO DE EDUCACIÓN MATEMÁTICA, IV, 2002, Chivilcoy, Argentina. Memorias... (CD-ROM)

GIL PÉREZ, D. (1993) La necesidad de innovaciones en la evaluación: enseñanza de las Ciencias y la Matemática. Valencia: Universidad de Valencia. Disponível em: <www.campus-oei.org $>$.

KEMPA, R. F. Students' learning difficulties in science. Causes and possible remedies. Enseñanza de las Ciencias, v. 9, n. p. 119-128, 1991.

LOMBANA, C. A. S. Metacognición. Cambio conceptual y enseñanza de las ciencias. Colombia: Ed. Didácticas Magisterio, 2003. 
MÉNDEZ, A. Evaluar para conocer, examinar para excluir. Madrid: Ediciones Morata S. L., 2001.

MOREIRA, M. La teoría del aprendizaje significativo de Ausubel. In: MOREIRA, M. (Ed.). Enfoques teóricos. Monografías sobre aprendizagem e ensino. Porto Alegre: Editora da UFRGS, 1997b.

MOREIRA, M. La teoría del desarrollo cognitivo de Piaget. In: MOREIRA, M. (Ed.). Enfoques teóricos. Monografías sobre aprendizagem e ensino. Porto Alegre: Editora da UFRGS, 1997a.

PEREZ, D. G. Relaciones entre conocimiento escolar y conocimiento científico. Investigación en la Escuela, v. 21, p. 17-32, 1994.

PÉREZ, M. G. Evaluación del aprendizaje en la enseñanza universitaria. Cuba: Universidad de La Habana, 2000.

RAYA, F. Los procesos de evaluación del aprendizaje presentes en las Cátedras de Botánica General en las Carreras de Ingeniería Agronómica del NOA. 2001. Tesis (Magíster) - Universidad Nacional de Tucumán, Tucumán, Argentina.

RAYA, F.; CUDMANI, L. C. Metaevaluación en Ciencias: una mirada retrospectiva para una investigación educativa. Revista de Enseñanza de la Física, Buenos Aires, v. 12, 2001.

ROBERTS, M. J.; ERDOS, G. Strategy selection and metacognition. Educational Psychology, v. 13, n. 3, p. 259-266, 1993.

SACRISTÁN, J. G.; GÓMEZ, A. P. Comprender y transformar la enseñanza. Madrid: Ediciones Morata, 1992.

SANZ, T. La planificación pedagógica de la enseñanza. La Habana, Cuba, 1991.

SCHMIDT, H. G. Fundamentos del aprendizaje basado sobre problemas: algunas notas explicativas. Medical Education, v. 27, p. 422-432, 1993.

SCHMIDT, H. G. La racionalidad detrás del ABP. In: SCHMIDT, H. G. Nuevas direcciones para la educación médica. New York: Springer Verlag, 1989. cap 8.

VARGAS, M. R. S.; CUDMANI, L. C. Estrategias Metacognitivas para la evaluación en Física. In: SIEF, 2006, Gualeguaychú, Argentina. Memorias... 


\section{Apéndice I}

\section{Actividad 1}

Resuelve la siguiente situación problemática.

I) Un cuerpo de $40 \mathrm{~N}$ está apoyado sobre un plano inclinado áspero que tiene $30^{\circ}$ de inclinación. Averiguar el valor de la fuerza de rozamiento para que el cuerpo baje con velocidad constante.

Contesta el siguiente cuestionario que te permitirá reflexionar sobre tus propios procesos de aprendizaje, sobre donde surgen tus dificultades y tus dudas y sobre qué cuestiones resultan valiosas y facilitadoras para resolver los problemas.

Tu respuesta será un aporte valioso para la enseñanza y el aprendizaje de la Física. La justificación de tus respuestas es lo más valioso para estos objetivos.

A) ¿Tuviste dificultad en la resolución de este problema? Marca la opción correcta.<smiles>[SiH3]C1CCC1</smiles>

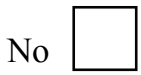

Si contestaste afirmativamente, sigue respondiendo el cuestionario

B) Tu dificultad en la resolución de este problema se encuentra en :

a) Interpretación del enunciado

b) Identificación de los datos.

c) Identificación de las fuerzas que actúan sobre el cuerpo

d) Aplicación de las leyes de la Dinámica

e) EL planteo de las ecuaciones

f) La resolución de las ecuaciones.

g) Otras

Justifica en cada caso tu respuesta.

C) Para superar tu o tus dificultades crees que debes: Marca la o las opciones que consideres más efectivas.

a) Pedir ayuda a un compañero para que te explique

b) Solicitar una nueva explicación al profesor

c) Resolver mayor cantidad de ejercicios

d) Cambiar tu forma de estudiar

e) No sabes como superar tu dificultad

f) Otras sugerencias

D) Consideras que estas reflexiones influirán favorablemente en el aprendizaje del tema? Porqué? 


\section{Actividad 2}

Resuelve la siguiente situación problemática:

II) Un cuerpo de $40 \mathrm{~N}$ está apoyado sobre un plano inclinado liso que tiene $30^{\circ}$ de inclinación. Al soltarlo, el cuerpo desciende. Averiguar el valor de la aceleración que adquiere.

Contesta el siguiente cuestionario que te permitirá reflexionar sobre tus propios procesos de aprendizaje, sobre donde surgen tus dificultades y tus dudas y sobre qué cuestiones resultan valiosas y facilitadoras para resolver los problemas.

Tu respuesta será un aporte valioso para la enseñanza y el aprendizaje de la Física. La justificación de tus respuestas es lo más valioso para estos objetivos.

A) ¿Tuviste dificultad en la resolución de este problema? Marca la opción correcta.
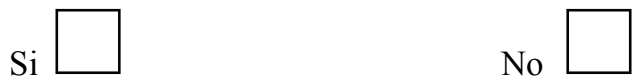

Si contestaste afirmativamente, sigue respondiendo el cuestionario

B) Tu dificultad en la resolución de este problema se encuentra en :

a) Interpretación del enunciado

b) Identificación de los datos.

c) Identificación de las fuerzas que actúan sobre el cuerpo

d) Aplicación de las leyes de la Dinámica

e) EL planteo de las ecuaciones

f) La resolución de las ecuaciones.

g) Otras

Justifica en cada caso tu respuesta.

C) Para superar tu o tus dificultades crees que debes: Marca la o las opciones que consideres más efectivas.

a) Pedir ayuda a un compañero para que te explique

b) Solicitar una nueva explicación al profesor

c) Resolver mayor cantidad de ejercicios

d) Cambiar tu forma de estudiar

e) No sabes como superar tu dificultad

f) Otras sugerencias

D) Consideras que estas reflexiones influirán en el aprendizaje de este tema? ¿Porqué? 